\title{
Outcome of ankle arthrodesis and total ankle replacement for ankle arthrosis in terms of gait variability
}

\author{
Swati Chopra ${ }^{1}$, Hossein Rouhani ${ }^{2}{ }^{3}$, Kevin Moerenhout ${ }^{1}$, J ulien Favre $^{2}$, Kamiar Aminian $^{2}$, Xavier \\ Crevoisier $^{1}$
}

1. Centre Hospitalier Universitaire Vaudois and University of Lausanne (CHUV), Department of Orthopaedic Surgery and Traumatology, Lausanne, Switzerland. 2. Ecole Polytechnique Fédérale de Lausanne (EPFL), Laboratory of Movement Analysis and Measurement, Lausanne, Switzerland. 3. Department of Mechanical Engineering, University of Alberta, Edmonton, Alberta, Canada

Correspondence: Swati Chopra. Address: Centre Hospitalier Universitaire Vaudois and University of Lausanne (CHUV), Site Hôpital Orthopédique, Service d'Orthopédie et de Traumatologie, Pierre-Decker 4, CH-1011 Lausanne, Switzerland. Email: swati.chopra@chuv.ch; schopra.research@gmail.com

Received: August 30, 2015

DOI : 10.5430/jbei.v2n1p31
Accepted: September 16, 2015

URL: http://dx.doi.org/10.5430/jbei.v2n1p31

\section{Abstract}

Background: Higher variability in gait parameters has been reported in patients' with arthrosis of hip and knee joints, leading to dynamic instability and increased risk of falling. Higher variability and gait instability has also been noted to persist months after surgery. Ankle arthrosis is likely similar, but information regarding this is lacking. The purpose of the study was to evaluate gait variability following ankle arthrosis and its surgical treatments, ankle arthrodesis and total ankle replacement.

Methods: Seventy participants, divided into controls, ankle arthrosis, ankle arthrodesis and total ankle replacement, performed gait assessment wearing 3-D inertial sensors through which gait parameters and the variability were compared among groups. Correlations between American Orthopedic Foot and Ankle score and Foot and Ankle Ability Measure score with the gait parameters and their variability were calculated.

Results: All three case groups reported gait variability similar to that of the controls. On the contrary, significant differences $(P<.01)$ were reported in several gait parameters when compared to the controls in all case groups. Furthermore, both clinical scores showed little to no correlation with gait variability and a good correlation was reported with gait parameters $(P<.0001)$.

Conclusions: The study did not find gait variability to be as reliable compared with gait parameters when assessing the outcome of ankle surgeries. Furthermore, among the gait parameters, walking speed showed a strong correlation with the patients' functional status and is confirmed as an important parameter for ankle arthrosis.

\section{Key words}

End stage ankle surgeries, American orthopaedic foot and ankle score, Foot and ankle ability measure, Outcome evaluation, Gait assessment 


\section{Introduction}

Gait variability describes the fluctuation in gait parameters from stride to stride and has been reported as a quantifiable indicator of walking function ${ }^{[1]}$. It is expressed by the coefficient of variance (CV \%) of spatiotemporal and kinematic parameters of gait. In a dynamic environment, gait variability plays a very important role in providing a stable and good functional mobility with time ${ }^{[1-3]}$. Hence, some variability in gait parameters is normal and important for stability and quality of movement. However, undesirably high or low variability in gait parameters due to a disturbance in gait regulating systems is not adaptive in nature and leads to instability, increasing the risk of fall ${ }^{[1,2]}$. Pathologic joint laxity or restriction leads to compensatory gait patterns with biomechanical deficits, produced by the surrounding structures. This leads to the abnormal increase or decreases in gait variability and reduced postural stability due to the loss of normal sensorimotor integrity ${ }^{[4]}$. The importance of gait variability in assessing gait stability and regularity has been proven in several studies ${ }^{[5,6]}$. Gait variability is also found to be more sensitive in assessing gait instability and fall risk compared to other methods ${ }^{[1,5]}$.

In hip and knee arthrosis, instability and increased risk of falling has been found to be associated with higher gait variability. As such, it is suggested as a meaningful factor in assessing post-surgical outcome ${ }^{[7-11]}$. Studies have shown the existence of adapted gait mechanics in end-stage ankle arthrosis patients ${ }^{[12]}$, which are also found to persist even after surgical correction of the disease ${ }^{[13-15]}$. Altered gait mechanics in ankle arthrosis, as in arthrosis of hip and knee joints, may result in reduced physical activity, gait instabilities and risk of fall ${ }^{[8,9]}$. It is, therefore, important to understand the relationship between gait variability and gait stability in ankle arthrosis and after its surgical corrections.

The present study aimed to assess gait variability-a marker of gait instability, gait irregularity and risk of fall in end stage ankle arthrosis and its two common surgical treatments: ankle arthrodesis and total ankle replacement. Participants gait were evaluated using an ambulatory measurement system. The working hypothesis of the study was that the gait parameters and their variability differ significantly in ankle arthrosis, ankle arthrodesis and total ankle replacement in comparison to the controls. Furthermore, the correlation between foot and ankle clinical scores and gait parameters along with their variability was assessed.

\section{Material and methods}

\subsection{Participants}

The study included 70 participants divided into four groups: 15 ankle arthrosis, 20 total ankle replacement, 15 ankle arthrodesis, and 20 healthy controls. Patients with unilateral isolated end stage ankle arthrosis, stages 3 and 4 according to Kellgren and Lawrence ${ }^{[16]}$, were included. The operated group consisted of patients with unilateral surgery (ankle arthrodesis or total ankle replacement), with no other pathology of the lower limbs, spine or other factors affecting gait. Inclusion criteria for healthy controls were the absence of any lower limb symptoms or pathology and or other factors affecting gait. All participants gave informed consent. A single surgeon performed both total ankle replacement and ankle arthrodesis surgeries. Patients' selection for ankle arthrodesis or total ankle replacement was nonrandomized and based purely on patients interest. For total ankle replacement surgeries, (Salto, Tornier ${ }^{\mathbb{R}}$, Montbonnot, FR), a mobile bearing implant with three components was used through an anterior approach. For ankle arthrodesis surgeries, internal fixation was performed through a lateral approach using 7.0 cannulated screws (Synthes ${ }^{\circledR}$, Oberdorf, $\mathrm{CH}$ ). The mean postoperative follow-up period for the two surgical groups for the gait assessment was 4.7 years ( \pm 2.7 years). Approval of the ethics commission of the University hospital was obtained.

\subsection{Assessment method}

Clinical assessment was performed using the hindfoot score AOFAS (American Orthopaedic Foot and Ankle Score) ${ }^{[17]}$ and the French version of daily activity sub score of the FAAM (Foot and Ankle Ability Measure) score ${ }^{[18]}$. The sports section of the FAAM was not included in the statistical comparison because most of our patients from all three groups had 
left the section incomplete. Gait assessment was performed using 3D inertial sensors, which were connected to an ambulatory data-logger (Physilog ${ }^{\circledR}$, BioAGM, CH). The sensors (3D accelerometers and gyroscopes) were attached to bony surfaces, minimizing the soft tissue artifact due to muscle contraction. The placement of the sensors was the same as in a previous validation study ${ }^{[15]}$. Participants were provided with custom made flat sandals that were available in various sizes. Based on the validated protocol, participants walked twice, at their preferred walking speed, along the 50-meter long hospital corridor ${ }^{[19]}$. Spatiotemporal and angular parameters of gait assessed include: cadence (step/min), stance time (gait cycle time [GCT\%]), inner stance event (load, foot-flat and push-off) ${ }^{[20]}$, stride (m), speed (m/sec), peak swing speed (PSS) $(\% / \mathrm{sec})$, toe-off pitch angle (TOP) $\left({ }^{\circ}\right)$ and heel-strike pitch angle (HSP) $\left({ }^{\circ}\right)$. The affected side was tested for the study groups and randomly selected side for the controls.

\subsection{Statistical analysis}

For each trial of $50 \mathrm{~m}$, the mean and standard deviation (SD) of each assessed parameter was estimated over all gait cycles after discarding the first three and last three gait cycles. After that, stride-to-stride variability was calculated using the coefficient of variation $(\mathrm{CV} \%=100 \times \mathrm{SD} /$ mean). Shapiro-Wilk test of normality was performed to test if the normal distribution exists in all groups for each assessed parameter. Results showed that most gait parameters and variability parameters were not normally distributed among the groups. Hence, robust non-parametric Wilcoxon rank sum test were performed to compare gait parameters and their variability between all four groups. Correlation between gait parameters and their gait variability with the clinical scores was calculated using Pearson's correlation coefficient. For all statistical analysis, the level of significance was set at $P<.01$ instead of $P<.05$. This is because with multiple comparisons between groups, a lower significance threshold could have a higher probability of false positives. However, for demographic and clinical score comparison the significant level was set at $P<.05$ as the multiple comparisons would not affect the outcome.

\section{Results}

\subsection{Demographics \& clinical score}

No significant difference was seen between the three case groups in age and body mass index (BMI). However, all three case groups were on average older and heavier $(P<.05)$ compared to the controls. A significantly higher $(P<.01)$ AOFAS score was reported in both surgical groups in comparison with the ankle arthrosis group. Furthermore, function sub score were found to be similar in ankle arthrosis and ankle arthrodesis groups. Lastly, the FAAM-ADL sub score showed improvement in both the operative groups in comparison to the ankle arthrosis group. However, the improvement was found to be significant only in total ankle replacement group $(P=.01)$ when compared with the ankle arthrosis group (see Table 1). Both the clinical scores showed a significantly reduced functional status in patients of the three case groups in comparison to the controls.

Table 1. Demographics and clinical scores of participants in control group and of patients, mean (SD)

\begin{tabular}{|c|c|c|c|c|}
\hline Physical characteristics & Control & Ankle arthrosis & Ankle arthrodesis & Total ankle replacement \\
\hline Age (years) & $59.3(8.9)$ & $65.8(9.8)^{*}$ & $64.2(9.3)^{*}$ & $63.6(9.3)^{*}$ \\
\hline BMI $\left(\mathrm{Kg} / \mathrm{m}^{2}\right)$ & $24.36(4.9)^{4 \uparrow 100}$ & $29.7(6.2)^{*}$ & $28.08(6.4)^{*}$ & $28.72(4.8)^{*}$ \\
\hline Sex & $16 \mathrm{~F} / 4 \mathrm{M}$ & $10 \mathrm{~F} / 5 \mathrm{M}$ & $9 \mathrm{~F} / 6 \mathrm{M}$ & $8 \mathrm{~F} / 12 \mathrm{M}$ \\
\hline AOFAS Total & $100(0)^{\dagger \uparrow \pi \infty}$ & $55(20)^{* \pi \infty \infty}$ & $70(11.5)^{\dagger *}$ & $81.5(20.7)^{\dagger^{*}}$ \\
\hline Pain & $40(0)^{\dagger \uparrow \infty \infty}$ & $20(20)^{* \pi / \infty}$ & $30(15)^{\dagger *}$ & $30(7.5)^{\dagger^{*}}$ \\
\hline Function & $50(0)^{\dagger+100}$ & $31(8)^{* \infty}$ & $31(4)^{* \infty}$ & $41(8.7)^{\dagger * \pi}$ \\
\hline Alignment & $10(0)^{\dagger \oplus \infty 0 \infty}$ & $5(2.5)^{* \pi \pi_{\infty}}$ & $10(0)^{\dagger^{* *}}$ & $10(0)^{\dagger^{*}}$ \\
\hline FAAM-ADL & $100(0)^{\dagger \uparrow \oplus_{\infty}}$ & $61.2(18.5)^{* \infty}$ & $68.75(17.6)^{*}$ & $79.8(17.4)^{* \dagger}$ \\
\hline
\end{tabular}

Note. ${ }^{*}$ indicated significance difference compared to controls, ${ }^{\dagger}$ represent significant difference compared to ankle arthrosis, ${ }^{\natural}$ indicates significant difference compared to ankle arthrodesis, ${ }^{\infty}$ represent significant difference compared to total ankle replacement $(P<.05)$. AOFAS: American orthopaedic foot and ankle society hindfoot score, FAAM-ADL: Foot and ankle ability measure score - activity of daily living section. 


\subsection{Spatiotemporal and angular parameters of gait}

Results of gait parameters for the four groups are given in Table 2. Comparing with the controls, the ankle arthrosis group showed significantly reduced cadence, load, push-off, stride, speed, PSS, HSP, TOP $(P<.01)$ and increased foot-flat duration $(P<.01)$. Similarly, ankle arthrodesis group showed significantly reduced cadence, push-off, stride, speed, peak swing speed, toe-off pitch angle $(P<.01)$ and increased foot-flat duration $(P<.01)$. Whilst, total ankle replacement group showed significantly reduced cadence, push-off, speed, TOP $(P<.01)$ and increased foot-flat duration $(P<.01)$.

Comparing with the AOA group, no significant difference was seen in the ankle arthrodesis group. However, total ankle replacement group showed a significantly increased cadence, stride, speed, peak swing speed, heel-strike pitch angle and toe-off pitch angle $(P<.01)$. Lastly, a comparison between total ankle replacement and ankle arthrodesis group showed a significantly increased cadence, stride, speed, peak swing speed and reduced toe-off pitch angle $(P<.01)$.

Table 2. Parameters of gait in controls and patients, mean (SD)

\begin{tabular}{|c|c|c|c|c|}
\hline Gait Parameters & Control & Ankle Arthrosis & Ankle Arthrodesis & Total Ankle Replacement \\
\hline Cadence & $117.6(1.3)^{\dagger \uparrow \infty \infty}$ & $96.56(16.5)^{* \infty}$ & $100.1(12.1)^{* \infty}$ & $109.3(9.4)^{\dagger \uparrow}$ \\
\hline Stance (GCT\%) & 58.47 (1.99) & $58.8(2.4)$ & $58.8(2.7)$ & $58.9(2.2)$ \\
\hline Load $(\mathrm{St} \%)$ & $12.1(3.3)^{\dagger}$ & $9.7(2.9)^{*}$ & $11.9(4.4)$ & $11.3(2.4)$ \\
\hline Foot-flat (St\%) & $52.5(5.9)^{\dagger \uparrow \infty}$ & $60.8(10.2)^{*}$ & $61.1(8.5)^{*}$ & $57.7(6.1)^{*}$ \\
\hline Push-off (St\%) & $35.5(4.87)^{\dagger \uparrow \infty}$ & $29.6(9.6)^{*}$ & $26.9(5.6)^{*}$ & $30.98(6.4)^{*}$ \\
\hline Stride (m) & $1.3(0.1)^{\dagger \uparrow}$ & $1.04(0.2)^{* \infty}$ & $1.04(0.18)^{* \infty}$ & $1.17(0.12)^{\dagger \uparrow}$ \\
\hline Speed $(\mathrm{m} / \mathrm{s})$ & $1.3(0.18)^{\dagger \oplus \infty \infty}$ & $0.84(0.27)^{* \infty}$ & $0.87(0.23)^{* \infty}$ & $1.08(0.2)^{*+\uparrow}$ \\
\hline $\operatorname{PSS}(\%)$ & $407.5(52.7)^{\dagger \oplus \pi}$ & $290.7(73.7)^{* \infty}$ & $306.4(47.4)^{* \infty}$ & $372.1(53.7)^{\dagger}$ \\
\hline $\operatorname{HSP}\left(^{\circ}\right)$ & $20.48(4.05)^{\dagger}$ & $16.7(5.2)^{* \infty}$ & $19(5.4)$ & $20(4.4)^{\dagger}$ \\
\hline TOP $\left({ }^{\circ}\right)$ & $-74.7(7.6)^{\dagger \oplus \infty}$ & $-55.1(10.2)^{* \infty}$ & $-53.5(6.9)^{* \infty}$ & $-63.5(8.6)^{*+\uparrow}$ \\
\hline
\end{tabular}

Note. "indicated significance difference compared to controls, ${ }^{\dagger}$ represent significant difference compared to ankle arthrosis, ${ }^{9}$ indicates significant difference compared to ankle arthrodesis, ${ }^{\infty}$ represent significant difference compared to total ankle replacement $(P<.01)$. PSS: peak swing speed, HSP: heel-strike pitch angle, TOP: toe-off pitch angle.

\subsection{Variability in parameters of gait}

Results of gait parameter variability are given in Table 3.

Table 3. Stride to stride variability (expressed by $\mathrm{CV} \%(100 \times \mathrm{std} / \mathrm{mean}))$ of gait parameter in controls and patients, mean (SD)

\begin{tabular}{lllll}
\hline Gait Parameter variability & Control & Ankle Arthrosis & Ankle Arthrodesis & Total Ankle Replacement \\
\hline Cadence & $3.2(1.1)^{\dagger}$ & $4.3(2.1)^{*}$ & $4.1(1.3)^{*}$ & $3.4(1.0)$ \\
Stance & $2.8(1.1)^{\dagger}$ & $3.8(2.0)^{* \infty}$ & $3.3(0.9)^{*}$ & $2.9(1.2)^{\dagger}$ \\
Load & $11.0(3.1)$ & $13.2(6.5)$ & $13.1(5.5)$ & $12.2(4.9)$ \\
Foot-flat & $6.7(2.4)^{\infty}$ & $6.3(4.7)$ & $5.6(2.1)$ & $5.68(3.5)^{*}$ \\
Push-off & $8.5(2.4)$ & $13.1(8.1)$ & $9.3(4.0)$ & $8.9(4.7)$ \\
Stride & $10.2(2.5)$ & $11.5(4.4)$ & $11.3(3.36)$ & $12.2(2.7)$ \\
Speed & $11.7(2.1)$ & $13.1(4.6)$ & $12(2.4)$ & $12.3(2.5)$ \\
PSS & $9.6(3.5)$ & $11.3(2.8)$ & $10.6(3.2)$ & $10.2(1.8)$ \\
HSP & $14.83 .3)$ & $18.2(9.5)$ & $15.4(5.8)$ & $14.9(3.2)$ \\
TOP & $10.3(3.7)$ & $12.3(3.7)$ & $11.1(3.3)$ & $10.7(2.6)$ \\
\hline
\end{tabular}

Note. ${ }^{*}$ indicated significance difference compared to controls, ${ }^{\dagger}$ represent significant difference compared to ankle arthrosis, ${ }^{\natural}$ indicates significant difference compared to ankle arthrodesis, ${ }^{\infty}$ represent significant difference compared to total ankle replacement $(P<.01)$. PSS: peak swing speed, HSP: heel-strike pitch angle, TOP: toe-off pitch angle. 
Compared to the controls gait variability was found to be significantly high in only cadence and stance in ankle arthrosis and ankle arthrodesis group $(P<.01)$, whilst in the total ankle replacement group a significantly reduced variability was reported only in foot-flat parameter. No significant difference was reported between the variability of ankle arthrosis and ankle arthrodesis group, however, the total ankle replacement group showed significantly low variability in comparison to ankle arthrosis in only the stance phase. Comparison between the two surgical groups showed no significant difference in gait variability. Furthermore, gender comparison was made because of the unequal distribution of males and females in each group, however, results showed no significant difference in the gait parameters or their variability.

\subsection{Correlation}

Results reported a strong correlation between AOFAS score and most gait parameters $(P<.0001)$ including cadence, push-off, stride, peak swing speed, toe-off pitch angle and speed (see Figure 1a), however, with respect to the variability in gait parameters a weak to negligible correlation was reported (see Figure 1b). Similarly, with FAAM score, gait parameters reported a strong correlation in most gait parameters including cadence, foot-flat, push-off, stride, PSS, TOP, HSP and speed $(P<.0001)$ (see Figure 1a), however, in relation to the variability only foot-flat showed above $30 \%$ of correlation (see Figure 1b). For a detailed understanding of the effect of demographics on gait variability, the correlation between gait variability and age, BMI was also assessed. No significant correlation was seen between age, BMI and gait parameters along with their variability. Lastly, as patients walked in their preferred walking speed which differs significantly among groups, the correlation between walking speed and other gait parameters along with their variability was assessed. The results showed moderate to strong correlation $(P<.0001)$ with most parameters except stance and load. While in relation to the variability in the parameters, walking speed showed weak to moderate correlation (see Figure 1c).
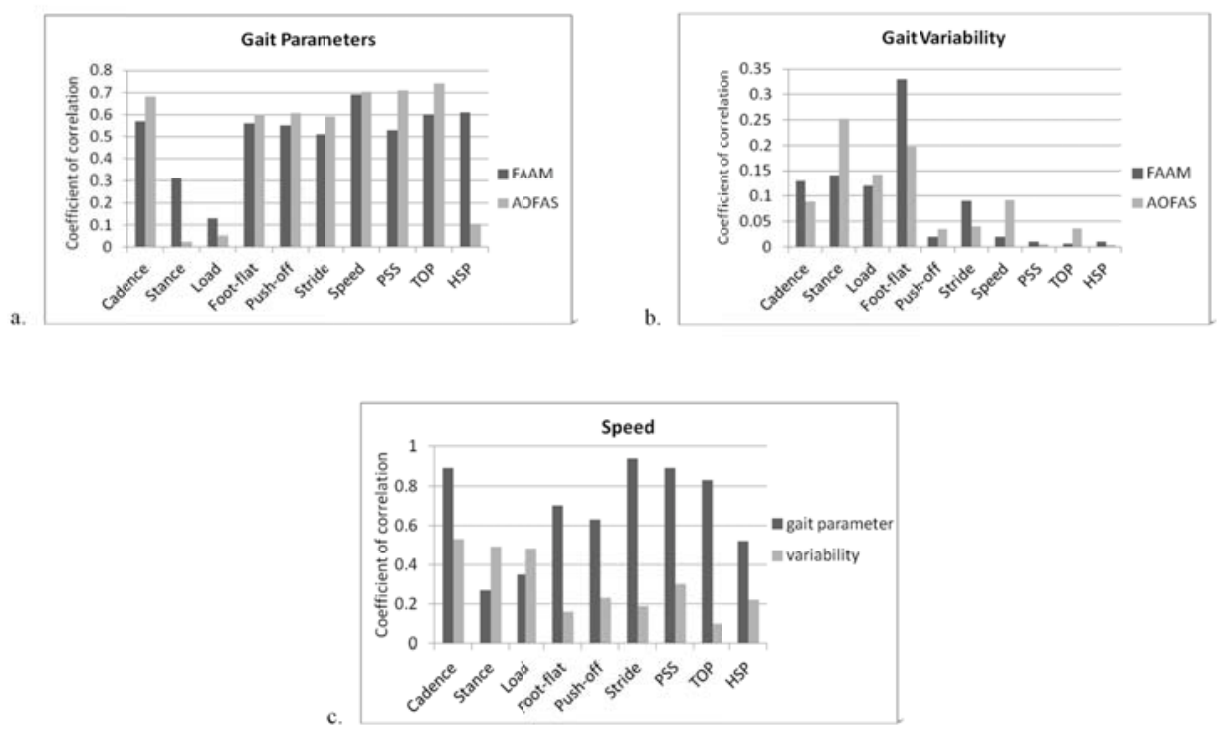

Figure 1. (a) Correlation between AOFAS and FAAM with the gait parameters, (b) Correlation between AOFAS and FAAM with the variability in gait parameters, (c) Correlation between speed and the gait parameters along with the gait variability.

\section{Discussions}

Studies have shown that dynamic instability can be associated with increased gait variability in patients with various medical conditions, including arthrosis of weight bearing joints like hip and knee, leading to an increased risk of fall $^{[5,9,21,22]}$. However, the extent of gait variability has not been assessed in ankle arthrosis and its surgical treatments. The study aimed to assess the variability in gait parameters in ankle arthrosis, ankle arthrodesis and total ankle replacement 
patients. The working hypothesis of the study was that the gait variability differs significantly in ankle arthrosis, ankle arthrodesis and total ankle replacement groups in comparison to the healthy controls. The study, however, did not report much difference in variability between the controls and the three case groups. These results gave a contrasting outcome in comparison to the studies assessing variability in arthrosis of hip and knee joints where the variability was reported to be higher in comparison to the controls ${ }^{[8,9]}$.

Comparing the variability in ten gait parameters between the controls and the three case groups, significant differences were reported for ankle arthrosis and ankle arthrodesis in only 2 out of 10 parameters and for total ankle replacement one out of 10 parameters. In contrast, comparing the gait parameters between the controls and the case groups reported significant differences in ankle arthrosis (9/10 gait parameters), ankle arthrodesis (7/10 gait parameters) and total ankle replacement (5/10 gait parameters) groups. Furthermore comparing variability between the ankle arthrosis group and the two surgical groups' significant difference was reported in only stance variability in total ankle replacement group, whilst gait parameter comparison showed significant difference in 6 out of 10 parameters in total ankle replacement group. Lastly, the comparison between the two surgical groups showed no difference in variability, whilst in gait parameters 5 out of 10 showed significant difference. As such, the study rejects the null hypothesis that gait variability is abnormally high following ankle arthrosis surgical treatments; ankle arthrodesis and total ankle replacement, in comparison to the healthy controls and as well as among each other.

In our study, the AOFAS and FAAM score results, representing the functional status of the patients, showed consistent results with previous studies ${ }^{[13,15,23,24]}$. A moderate to strong correlation was reported between both the clinical scores and the gait parameters in comparison to their variability. This suggests that the gait parameters relates better with the clinical status of the patient than their variability. On the contrary, the clinical score for knee had shown a strong correlation with the variability of knee motion in severe knee arthrosis ${ }^{[9]}$. No clear correlation was seen between age and gait parameters and or variability. The reason could be the patients' age (average 65 years), which is considered relatively early for the initiation of the age related general neuromuscular deterioration ${ }^{[3,5]}$. Furthermore, BMI also showed weak correlation with the gait variability, similar to the previous study assessing gait variability in knee arthrosis ${ }^{[9]}$.

Arthrosis of hip and knee joints is mostly due to idiopathic degeneration and tends to appear at an older age in comparison to ankle arthrosis, $70 \%$ of cases have a post-traumatic origin and most patients develop the disease at a relatively young age $^{[23]}$. Elderly patients along with the existing insufficient neuromuscular control due to hip or knee degeneration may be the reason behind the high incidence of fall during the early postoperative phase ${ }^{[7]}$. Furthermore, the major postural muscles of the lower limbs including quadriceps, gluteus, and hamstrings are affected (wasting and or compensatory over activity) with arthrosis of knee and hip joints ${ }^{[25-27]}$, affecting the balance and increasing the risk of fall. Muscles affected significantly in end stage ankle arthrosis include medial soleus and only fatty degeneration was reported in other muscles ${ }^{[28]}$. This may be the reason postural stability remains preserved in ankle arthrosis as well as why gait variability parameters are not as important in ankle arthrosis as they are in arthrosis of other weight bearing joints.

Strength of the study is the large cohort size, including ankle arthrosis, ankle arthrodesis and total ankle replacement patients, which revealed more precise results of comparison between the four study groups. The ambulatory gait analysis system used in the study also allowed measurement of several strides at a time, in an open environment-necessary for an accurate assessment of gait variability ${ }^{[3]}$. The study, however, also has some limitations. In FAAM, only ADL section of the score was utilized, instead of the complete score (ADL + sports). However, the purpose of the score was to find the extent of the correlation between functional status and gait variability. The results reported utilization of the FAAM-ADL was also conclusive irrespective of the inclusion of the sports section results. It is important to note that one cannot assume causality of the defined outcome of either of the surgical groups based purely on surgical intervention due to a lack of pre-operative data. On average there was a difference in demographics of controls compared to the study groups but our results showed no effect of age or BMI on the outcome of variability. Furthermore, the difference of 5 years between the 
controls and the case groups would not affect the gait results so significantly as to alter the outcome, and hence, this was considered to have little impact on the conclusions.

In conclusion, the study found that, unlike in hip and knee joint problems, gait variability is not an important parameter as compared to other parameters of gait for ankle joint pathologies. The study found that gait variability in ankle arthrosis, ankle arthrodesis and total ankle replacement patients were comparable to controls. However, gait parameters in all three case groups were found to be significantly different. As a result, the study found no additional information in patients' status utilizing gait variability parameters. The study, therefore, concludes that there is no evidence for basing the outcome assessment of an ankle arthrosis surgery on gait variability.

\section{References}

[1] Hausdorff JM. Gait variability: methods, modeling and meaning. J Neuroeng Rehabil. 2005; 2: 19. PMid:16033650. http://dx.doi.org/10.1186/1743-0003-2-19

[2] Stergiou N, Decker LM. Human movement variability, nonlinear dynamics, and pathology: is there a connection? Hum Mov Sci. 2011; 30(5): 869-88. PMid:21802756. http://dx.doi.org/10.1016/j.humov.2011.06.002

[3] Hausdorff JM. Gait dynamics, fractals and falls: finding meaning in the stride-to-stride fluctuations of human walking. Hum Mov Sci. 2007; 26(4): 555-89. PMid:17618701. http://dx.doi.org/10.1016/j.humov.2007.05.003

[4] Hurley MV, Scott DL, Rees J, et al. Sensorimotor changes and functional performance in patients with knee osteoarthritis. Ann Rheum Dis. 1997; 56(11): 641-8. PMid:9462165. http://dx.doi.org/10.1136/ard.56.11.641

[5] Hausdorff, JM, Edelberg HK, Mitchell SL, et al. Increased gait unsteadiness in community-dwelling elderly fallers. Arch Phys Med Rehabil. 1997; 78(3): 278-83. http://dx.doi.org/10.1016/S0003-9993(97)90034-4

[6] Sheridan PL, Hausdorff JM. The role of higher-level cognitive function in gait: executive dysfunction contributes to fall risk in Alzheimer's disease. Dement Geriatr Cogn Disord. 2007; 24(2): 125-37. PMid:17622760. http://dx.doi.org/10.1159/000105126

[7] Jorgensen CC, Kehlet H. Fall-related admissions after fast-track total hip and knee arthroplasty - cause of concern or consequence of success? Clin Interv Aging. 2013; 8: 1569-77. PMid:24348026. http://dx.doi.org/10.2147/CIA.S52528

[8] Kiss RM. Effect of walking speed and severity of hip osteoarthritis on gait variability. J Electromyogr Kinesiol. 2010; 20(6): 1044 -51. PMid:20822918. http://dx.doi.org/10.1016/j.jelekin.2010.08.005

[9] Kiss RM. Effect of severity of knee osteoarthritis on the variability of gait parameters. J Electromyogr Kinesiol. 2011; 21(5): 695 -703. PMid:21840223. http://dx.doi.org/10.1016/j.jelekin.2011.07.011

[10] McClelland JA, Webster KE, Feller JA. Variability of walking and other daily activities in patients with total knee replacement. Gait Posture. 2009; 30(3): 288-95. PMid:19616951. http://dx.doi.org/10.1016/j.gaitpost.2009.05.015

[11] Van den Akker-Scheek I, Stevens M, Bulstra SK, et al. Recovery of gait after short-stay total hip arthroplasty. Arch Phys Med Rehabil. 2007; 88(3): 361-7. PMid:17321830. http://dx.doi.org/10.1016/j.apmr.2006.11.026

[12] Segal AD, Shofer J, Hahn ME, et al. Functional limitations associated with end-stage ankle arthritis. J Bone Joint Surg Am. 2012; 94(9): 777-83. PMid:22552666. http://dx.doi.org/10.2106/JBJS.K.01177

[13] Chopra S, Rouhani H, Assal M, et al. Outcome of unilateral ankle arthrodesis and total ankle replacement in terms of bilateral gait mechanics. J Orthop Res. 2014; 32(3): 377-84. PMid:24243755. http://dx.doi.org/10.1002/jor.22520

[14] Horisberger M, Hintermann B, Valderrabano V. Alterations of plantar pressure distribution in posttraumatic end-stage ankle osteoarthritis. Clin Biomech (Bristol, Avon). 2009; 24(3): 303-7. PMid:19150745.

http://dx.doi.org/10.1016/j.clinbiomech.2008.12.005

[15] Rouhani H, Favre J, Aminian K, et al. Multi-segment foot kinematics after total ankle replacement and ankle arthrodesis during relatively long-distance gait. Gait Posture. 2012; 36(3): 561-6. PMid:22763319. http://dx.doi.org/10.1016/j.gaitpost.2012.05.010

[16] Kellgren, JH, Lawrence JS. Radiological Assessment of Osteo-Arthrosis. Annals of the Rheumatic Diseases. 1957; 16(4): 494502. PMid:13498604. http://dx.doi.org/10.1136/ard.16.4.494

[17] Hunt KJ, Hurwit D. Use of patient-reported outcome measures in foot and ankle research. J Bone Joint Surg Am. 2013; 95(16): e118 (1-9).

[18] Borloz S, Crevoisier X, Deriaz O, et al. Evidence for validity and reliability of a French version of the FAAM. BMC Musculoskelet Disord. 2011; 12: 40. PMid:21303520. http://dx.doi.org/10.1186/1471-2474-12-40

[19] Rouhani H, Favre J, Crevoisier X, et al. Measurement of multi-segment foot joint angles during gait using a wearable system. J Biomech Eng. 2012; 134(6): 061006. PMid:22757503. http://dx.doi.org/10.1115/1.4006674 
[20] Mariani B, Rouhani H, Crevoisier X, et al. Quantitative estimation of foot-flat and stance phase of gait using foot-worn inertial sensors. Gait Posture. 2013; 37(2): 229-34. PMid:22877845. http://dx.doi.org/10.1016/j.gaitpost.2012.07.012

[21] Coelho FG, Stella F, de Andrade LP, et al. Gait and risk of falls associated with frontal cognitive functions at different stages of Alzheimer's disease. Neuropsychol Dev Cogn B Aging Neuropsychol Cogn. 2012; 19(5): 644-56. PMid:22360785. http://dx.doi.org/10.1080/13825585.2012.661398

[22] Roemmich RT, Nocera JR, Vallabhajosula S, et al. Spatiotemporal variability during gait initiation in Parkinson's disease. Gait Posture. 2012; 36(3): 340-3. PMid:22543093. http://dx.doi.org/10.1016/j.gaitpost.2012.01.018

[23] Thomas R, Daniels TR, Parker K. Gait analysis and functional outcomes following ankle arthrodesis for isolated ankle arthritis. J Bone Joint Surg Am. 2006; 88(3): 526-35. PMid:16510818. http://dx.doi.org/10.2106/JBJS.E.00521

[24] Dalat F, Trouillet F, Fessy MH, et al. Comparison of quality of life following total ankle arthroplasty and ankle arthrodesis: Retrospective study of 54 cases. Orthop Traumatol Surg Res. 2014; 100(7): 761-6. PMid:25306302. http://dx.doi.org/10.1016/j.otsr.2014.07.018

[25] Dwyer MK, Stafford K, Mattacola CG, et al. Comparison of gluteus medius muscle activity during functional tasks in individuals with and without osteoarthritis of the hip joint. Clin Biomech (Bristol, Avon). 2013; 28(7): 757-61. PMid:23911109. http://dx.doi.org/10.1016/j.clinbiomech.2013.07.007

[26] Saleh KJ, Lee LW, Gandhi R, et al. Quadriceps strength in relation to total knee arthroplasty outcomes. Instr Course Lect. 2010; 59: 119-30. PMid:20415375.

[27] Rutherford DJ, Hubley-Kozey CL, Stanish WD. Changes in knee joint muscle activation patterns during walking associated with increased structural severity in knee osteoarthritis. J Electromyogr Kinesiol. 2013; 23(3): 704-11. PMid:23357547. http://dx.doi.org/10.1016/j.jelekin.2013.01.003

[28] Wiewiorski M, Dopke K, Steiger C, et al. Muscular atrophy of the lower leg in unilateral post traumatic osteoarthritis of the ankle joint. Int Orthop. 2012; 36(10): 2079-85. PMid:22722542. http://dx.doi.org/10.1007/s00264-012-1594-6 